(C) 2007 IEEE. Reprinted, with permission, from Youguang Guo, An Improved Multiquadric Collocation Method for 3D Electromagnetic Problems . Magnetics, IEEE Transactions on (Volume:43, Issue: 4 ), 2007. This material is posted here with permission of the IEEE. Such permission of the IEEE does not in any way imply IEEE endorsement of any of the University of Technology, Sydney's products or services. Internal or personal use of this material is permitted. However, permission to reprint/republish this material for advertising or promotional purposes or for creating new collective works for resale or redistribution must be obtained from the IEEE by writing to pubs-permissions@ieee.org. By choosing to view this document, you agree to all provisions of the copyright laws protecting it 


\title{
An Improved Multiquadric Collocation Method for 3-Dimensional Electromagnetic Problems
}

\author{
Yong Zhang, K.R. Shao, Youguang Guo, D.X. Xie and J.D. Lavers, Fellow, IEEE
}

Abstract-- The multiquadric radial basis function method (MQ RBF or, simply, MQ) developed recently is a truly meshless collocation method with global basis functions. It was introduced for solving many 1D and 2D PDEs, including linear and nonlinear problems. However, few works are found for electromagnetic PDEs, even for 3D problems. This paper presents an improved MQ collocation method for 3D electromagnetic problems. Numerical results show a considerable improvement in accuracy over the traditional MQ collocation method, although both methods are direct collocation method with exponential convergence.

Index Terms-Multiquadric, meshless method, 3D problems.

\section{INTRODUCTION}

$\mathbf{E}$ LECTROMAGNETIC problems in 3D domains are much more complex than that in lower dimensions due to its increment on the computational cost and difficulties in constructing the domain discretization. The FEM presents characteristics that permit to take into account different kind of materials and model geometrically complex domains. Howover, a good quality mesh procedure is needed in order to get much more accurate results. This procedure must correspondingly increase much of the computational cost, even for problems needed remeshing in moving geometry or shape optimization.

Meshless methods, developed in the last decade, form a new class of numerical techniques of which the main objective is to overcome the limitations imposed by traditional mesh structured methods. This type of methods uses a set of nodes distributed in solving domain instead of traditional elements [1]. Meshless techniques are divided into two classes: those based on collocations and those based on weak forms. The first class is truly meshless method and does not require a mesh structure or a numerical integration procedure. It is insensitive to spatial dimension, considering only a cloud of nodes for the spatial discretization of both the solving domain and the boundary [2].

Radial basis function (RBF) method, as a truly meshless method for approximating the solutions of PDEs, has drawn much of the attention of many researchers in science and

This work was supported by National Natural Science Foundation of China under Grant No.50477045 and SRPDP under Grant 20030478063.

Yong Zhang and K.R. Shao are with the College of Electrical \& Electronic Engineering, Huazhong University of Science and Technology, Wuhan 430074 P.R. China. (E-mail: krshao@hust.edu.cn)

Y. Guo is with the Faculty of Engineering, University of Technology, Sydney, NSW 2007, Australia (e-mail: youguang@eng.uts.edu.au).

D.X. Xie is with the Department of Electrical Engineering, Shenyang University of Technology, Shenyang 110023 P.R.China.

J.D. Lavers is with the Department of Electrical \& Computer Engineering, University of Toronto, Toronto, M5S 3G4, Canada. engineering. In this class of truly RBF meshless methods, the multiquadric RBF (MQ) method is ranked the best based on its high accuracy, ease of implementation, good visual aspect, and low execution time and storage requirements [3,4]. MQ method is only dependent on spatial coordinates. This characteristic can easily treat 3D electromagnetic problems, even for static and quasi-static electromagnetic problems, including linear or nonlinear Laplace and Poisson problems.

In this paper, an improved MQ method is proposed for 3D electromagnetic Laplace and Poisson problems. It is achieved by adding a set of nodes (which can lie inside or outside of the domain) adjacent to the boundary and, correspondingly, add an additional set of collocation equations obtained via collocation of the PDE on the boundary [5]. Numerical results show a considerable improvement in accuracy over the traditional MQ method, although both methods are direct collocation method with exponential convergence.

\section{Multiquadric Collocation Method}

The multiquadric collocation method belongs to RBF type methods. Consider a set of nodes $\mathbf{x}_{1}, \mathbf{x}_{2}, \cdots, \mathbf{x}_{N} \in \mathbf{R}^{n}$ (Fig.1).

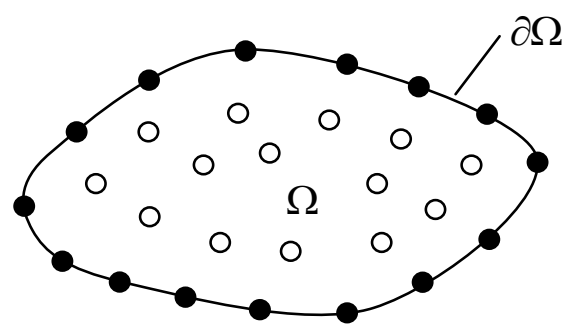

Fig.1 2D nodes diagram

The RBF centered at $\mathbf{x}_{j}$ is defined as

$$
\varphi_{j}(\mathbf{x}) \equiv \varphi\left(\left\|\mathbf{x}-\mathbf{x}_{j}\right\|, c\right) \in \mathbf{R}^{n}, j=1,2, \cdots, N
$$

where $\left\|\mathbf{x}-\mathbf{x}_{j}\right\|$ is the Euclidian norm. The RBF-MQ is

$$
\varphi_{j}(\mathbf{x})=\left(\left\|\mathbf{x}-\mathbf{x}_{j}\right\|+c^{2}\right)^{1 / 2}
$$

where $c$ is a shape parameter, in 2D space, $\mathbf{x}=(x, y)$ and in 3D space, $\mathbf{x}=(x, y, z)$. Let $\Omega \subset \mathbf{R}^{n}$, we consider a linear elliptic boundary value problem of the form

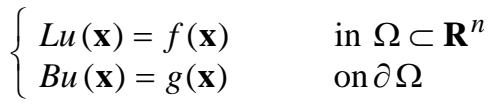

where $\Omega$ is a bounded domain with the boundary $\partial \Omega, L$ is a linear elliptic partial differential operator, and $B$ is a boundary operator. We use $N$ distinct nodes in and on the boundary of $\Omega$, of which $\left\{\mathbf{x}_{j}, j=1, \cdots N_{I}\right\}$ are interior 
nodes and $\left\{\mathbf{x}_{j}, j=N_{I}+1, \cdots N\right\}$ are boundary points. We look for the approximate solution $u_{h}(\mathbf{x})$ to (3) in the form

$$
u_{h}(\mathbf{x})=\sum_{j=1}^{N} a_{j} \varphi\left(\left\|\mathbf{x}-\mathbf{x}_{j}\right\|, c\right)=\sum_{j=1}^{N} a_{j} \varphi_{j}(\mathbf{x})
$$

where $\mathbf{a}=\left[a_{1}, \cdots, a_{N}\right]$ are unknown coefficients to be determined, $\varphi_{j}(\mathbf{x})$ is MQ RBF. Substituting $u_{h}(\mathbf{x})$ into (3) and using collocation at the $N$ nodes, we can get the finite dimensional problem

$$
\begin{aligned}
& \sum_{j=1}^{N} a_{j} L\left(\varphi_{j}\left(\mathbf{x}_{i}\right)\right)=f\left(\mathbf{x}_{i}\right), i=1, \cdots N_{I} \\
& \sum_{j=1}^{N} a_{j} B\left(\varphi_{j}\left(\mathbf{x}_{i}\right)\right)=g\left(\mathbf{x}_{i}\right), i=N_{I}+1, \cdots N
\end{aligned}
$$

This corresponds to the system of equations with a coefficient matrix, its matrix form and solution are

$$
\begin{gathered}
{\left[\begin{array}{l}
\mathbf{L}(\varphi) \\
\mathbf{B}(\varphi)
\end{array}\right][\mathbf{a}]=\left[\begin{array}{l}
\mathbf{f} \\
\mathbf{g}
\end{array}\right]} \\
{[\mathbf{a}]=\left[\begin{array}{l}
\mathbf{L}(\varphi) \\
\mathbf{B}(\varphi)
\end{array}\right]^{-1}\left[\begin{array}{l}
\mathbf{f} \\
\mathbf{g}
\end{array}\right]}
\end{gathered}
$$

In (6), we have

$$
\begin{aligned}
\mathbf{L}(\varphi) & =\left[\begin{array}{ccc}
L\left(\varphi_{1}\left(\mathbf{x}_{1}\right)\right) & \cdots & L\left(\varphi_{N}\left(\mathbf{x}_{1}\right)\right) \\
\vdots & \vdots & \vdots \\
L\left(\varphi_{1}\left(\mathbf{x}_{N I}\right)\right) & \cdots & L\left(\varphi_{N}\left(\mathbf{x}_{N I}\right)\right)
\end{array}\right] \\
\mathbf{B}(\varphi) & =\left[\begin{array}{ccc}
B\left(\varphi_{1}\left(\mathbf{x}_{N I+1}\right)\right) & \cdots & B\left(\varphi_{N}\left(\mathbf{x}_{N I+1}\right)\right) \\
\vdots & \vdots & \vdots \\
B\left(\varphi_{1}\left(\mathbf{x}_{N}\right)\right) & \cdots & B\left(\varphi_{N}\left(\mathbf{x}_{N}\right)\right)
\end{array}\right]
\end{aligned}
$$

MQ is only dependent on spatial coordinates, namely distributed nodes, it has no relationship with any mesh procedure, and this characteristic can make its procedures for solving PDE problems simple.

\section{IMPROVED MQ COLLOCATION METHOD}

\section{A. Improved truly MQ collocation method}

The improved truly MQ collocation method is achieved by adding a set of nodes (which can lie inside or outside of the domain) adjacent to the boundary and, correspondingly, adds and additional set of collocation equations obtained via collocation of the PDE on the boundary. Due to this change, the nodes in the domain and on the boundary are regarded as interior nodes, and the nodes outside of the domain are regarded as boundary nodes.

We still consider a set of nodes $\mathbf{x}_{1}, \mathbf{x}_{2}, \cdots, \mathbf{x}_{N} \in \mathbf{R}^{n}$ for the above linear elliptic boundary value problem in (3), in which $\left\{\mathbf{x}_{j}, j=1, \cdots N_{I}\right\}$ and $\left\{\mathbf{x}_{j}, j=N_{I}+1, \cdots N_{B}\right\}$ are interior and boundary nodes respectively, $\left\{\mathbf{x}_{j}, j=N_{B}+1, \cdots N\right\}$ are nodes adjacent to the boundary (Fig.2). We look for the approximate solution $u_{h}(\mathbf{x})$ to (3)

$$
u_{h}(x)=\sum_{j=1}^{N} a_{j} \varphi\left(\left\|\mathbf{x}-\mathbf{x}_{j}\right\|, c\right)=\sum_{j=1}^{N} a_{j} \varphi_{j}(\mathbf{x})
$$

where $\mathbf{a}=\left[a_{1}, \cdots, a_{N}\right]$ are unknown coefficients to be determined, $\varphi_{j}(\mathbf{x})$ is MQ RBF. Substituting $u_{h}(\mathbf{x})$ into (3) and using collocation at the $N$ nodes, we can get

$$
\begin{aligned}
& \sum_{j=1}^{N} a_{j} L\left(\varphi_{j}\left(\mathbf{x}_{i}\right)\right)=f\left(\mathbf{x}_{i}\right), i=1, \cdots, N_{I}, \cdots, N_{B} \\
& \sum_{j=1}^{N} a_{j} B\left(\varphi_{j}\left(\mathbf{x}_{i}\right)\right)=g\left(\mathbf{x}_{i}\right), i=N_{B}+1, \cdots N
\end{aligned}
$$

This corresponds to the system of equations with a coefficient matrix. Its matrix form and solution are the same to (6), but the elements of the matrix become to

$$
\begin{aligned}
\mathbf{L}(\varphi) & =\left[\begin{array}{ccc}
L\left(\varphi_{1}\left(\mathbf{x}_{1}\right)\right) & \cdots & L\left(\varphi_{N}\left(\mathbf{x}_{1}\right)\right) \\
\vdots & \vdots & \vdots \\
L\left(\varphi_{1}\left(\mathbf{x}_{N_{B}}\right)\right) & \cdots & L\left(\varphi_{N}\left(\mathbf{x}_{N_{B}}\right)\right)
\end{array}\right] \\
\mathbf{B}(\varphi) & =\left[\begin{array}{ccc}
B\left(\varphi_{1}\left(\mathbf{x}_{N_{B}+1}\right)\right) & \cdots & B\left(\varphi_{N}\left(\mathbf{x}_{N_{B}+1}\right)\right) \\
\vdots & \vdots & \vdots \\
B\left(\varphi_{1}\left(\mathbf{x}_{N}\right)\right) & \cdots & B\left(\varphi_{N}\left(\mathbf{x}_{N}\right)\right)
\end{array}\right]
\end{aligned}
$$

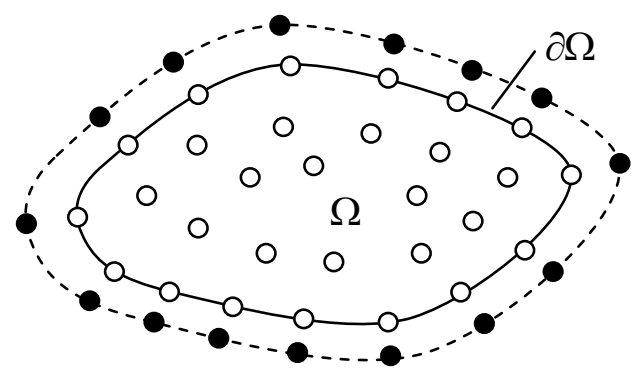

Fig.2 Domain and fictitious boundary nodes diagram

\section{B. Improved $M Q$ collocation method with polynomial basis}

In improved MQ collocation method, the approximate solution $\tilde{u}_{h}(x)$ to (3) can be rewritten as

$$
\tilde{u}_{h}(x)=\sum_{j=1}^{N} a_{j} \varphi_{j}(\mathbf{x})+\sum_{k=1}^{m} b_{k} p_{k}(\mathbf{x})
$$

Where $\mathbf{a}=\left[a_{1}, \cdots, a_{N}\right]$ and $\mathbf{b}=\left[b_{1}, \cdots, b_{m}\right]$ are unknown coefficients to be determined, $\varphi_{j}(\mathbf{x})$ and $p_{k}(\mathbf{x})$ are MQ RBF and polynomials respectively. The following constraint condition should also be satisfied in order to get unique solution:

$$
\sum_{j=1}^{N} a_{j} p_{k}\left(\mathbf{x}_{j}\right)=0, k=1,2, \cdots, m
$$

Substituting (11) and (12) into the linear elliptic boundary value problem described as (3), we also can get the finite dimensional problem as

$$
\begin{aligned}
& \sum_{j=1}^{N} a_{j} L\left[\varphi_{j}\left(\mathbf{x}_{i}\right)\right]+\sum_{k=1}^{m} b_{j} L\left[p_{k}\left(\mathbf{x}_{i}\right)\right]=f\left(\mathbf{x}_{i}\right), i=1, \cdots N_{B} \\
& \sum_{j=1}^{N} a_{j} B\left(\varphi_{j}\left(\mathbf{x}_{i}\right)\right)+\sum_{k=1}^{m} b_{j} B\left(p_{k}\left(\mathbf{x}_{i}\right)\right)=g\left(\mathbf{x}_{i}\right), i=N_{B}+1, \cdots N
\end{aligned}
$$


Combining (13) with (12), the matrix form of (6) can be rewritten as

$$
\begin{gathered}
{\left[\begin{array}{cc}
\mathbf{L}(\varphi) & \mathbf{L}(p) \\
\mathbf{B}(\varphi) & \mathbf{B}(p) \\
\mathbf{P}^{T} & \mathbf{0}
\end{array}\right]\left[\begin{array}{l}
\mathbf{a}_{1} \\
\mathbf{a}_{2} \\
\mathbf{b}
\end{array}\right]=\left[\begin{array}{l}
\mathbf{f} \\
\mathbf{g} \\
\mathbf{0}
\end{array}\right]} \\
{\left[\begin{array}{l}
\mathbf{a}_{1} \\
\mathbf{a}_{2} \\
\mathbf{b}
\end{array}\right]=\left[\begin{array}{cc}
\mathbf{L}(\varphi) & \mathbf{L}(p) \\
\mathbf{B}(\varphi) & \mathbf{B}(p) \\
\mathbf{P}^{T} & \mathbf{0}
\end{array}\right]^{-1}\left[\begin{array}{l}
\mathbf{f} \\
\mathbf{g} \\
\mathbf{0}
\end{array}\right]}
\end{gathered}
$$

Where

$$
\begin{aligned}
& \mathbf{L}(\varphi)=\left[\begin{array}{ccc}
L\left[\varphi_{1}\left(\mathbf{x}_{1}\right)\right] & \cdots & L\left[\varphi_{N}\left(\mathbf{x}_{1}\right)\right] \\
\vdots & \cdots & \vdots \\
L\left[\varphi_{1}\left(\mathbf{x}_{N_{B}}\right)\right] & \cdots & L\left[\varphi_{N}\left(\mathbf{x}_{N_{B}}\right)\right]
\end{array}\right] \\
& \mathbf{L}(p)=\left[\begin{array}{ccc}
L\left[p_{1}\left(\mathbf{x}_{1}\right)\right] & \cdots & L\left[p_{m}\left(\mathbf{x}_{1}\right)\right] \\
\vdots & \cdots & \vdots \\
L\left[p_{1}\left(\mathbf{x}_{N_{B}}\right)\right] & \cdots & L\left[p_{m}\left(\mathbf{x}_{N_{B}}\right)\right]
\end{array}\right] \\
& \mathbf{B}(\varphi)=\left[\begin{array}{ccc}
B\left[\varphi_{1}\left(\mathbf{x}_{N_{B}+1}\right)\right] & \cdots & B\left[\varphi_{N}\left(\mathbf{x}_{N_{B}+1}\right)\right] \\
\vdots & \cdots & \vdots \\
B\left[\varphi_{1}\left(\mathbf{x}_{N}\right)\right] & \cdots & B\left[\varphi_{N}\left(\mathbf{x}_{N}\right)\right]
\end{array}\right] \\
& \mathbf{B}(p)=\left[\begin{array}{ccc}
B\left[p_{1}\left(\mathbf{x}_{N_{B}+1}\right)\right] & \cdots & B\left[p_{m}\left(\mathbf{x}_{N_{B}+1}\right)\right] \\
\vdots & \cdots & \vdots \\
B\left[p_{1}\left(\mathbf{x}_{N}\right)\right] & \cdots & B\left[p_{m}\left(\mathbf{x}_{N}\right)\right]
\end{array}\right] \\
& \mathbf{P}^{T}=\left[\begin{array}{ccc}
p_{1}\left(\mathbf{x}_{1}\right) & \cdots & p_{1}\left(\mathbf{x}_{N}\right) \\
\vdots & \cdots & \vdots \\
p_{m}\left(\mathbf{x}_{1}\right) & \cdots & p_{m}\left(\mathbf{x}_{N}\right)
\end{array}\right] \\
& {\left[\mathbf{a}_{1} \mathbf{a}_{2}\right]^{T}=[\mathbf{a}]^{T}=\left[a_{1}, a_{2}, \cdots, a_{N}\right]} \\
& {[\mathbf{f}]^{T}=\left[f\left(\mathbf{x}_{1}\right), f\left(\mathbf{x}_{2}\right), \cdots, f\left(\mathbf{x}_{N_{B}}\right)\right]} \\
& {[\mathbf{g}]^{T}=\left[g\left(\mathbf{x}_{N_{B}+1}\right), g\left(\mathbf{x}_{N_{B}+2}\right), \cdots, g\left(\mathbf{x}_{N}\right)\right]}
\end{aligned}
$$

For RBF-MQ in 3D Cartesian coordinate, we have

$$
\begin{aligned}
& \varphi_{j}(\mathbf{x})=\sqrt{r_{j}^{2}+c^{2}} \\
& r_{j}=\sqrt{\left(x-x_{j}\right)^{2}+\left(y-y_{j}\right)^{2}+\left(z-z_{j}\right)^{2}} \\
& \frac{\partial \varphi_{j}(\mathbf{x})}{\partial \zeta}=\frac{\zeta-\zeta_{j}}{\left(r_{j}^{2}+c^{2}\right)^{1 / 2}}, \zeta=x, y, z \\
& \nabla^{2} \varphi_{j}(\mathbf{x})=\frac{2 r_{j}^{2}+3 c^{2}}{\left(r_{j}^{2}+c^{2}\right)^{3 / 2}}
\end{aligned}
$$

\section{NUMERICAL EXAMPLES}

In order to evaluate the effectivity and accuracy of the proposed method, a 3D problem with analytical solution is analyzed here. Suppose a cubical box (scale size $L=1.0$ ) with perfect conduct wall as shown in Fig.3, its up wall does not connect with the other five walls. The boundary condition is $V=1$ to the up wall and $V=0$ to others. This problem is an excellent benchmark because it not only has analytical solution but also has strong variation of the electrostatic potential in the four up corners.

To solve this problem with the proposed method, nearly uniform nodes are distributed in the solving domain and the outer boundary. Fig. 4 is a cross section of the nodes distribution for the benchmark problem at $y=0.6$, there are the same number of nodes distributed outside of the boundary to those distributed on the real boundary.

To compare the numerical results between the improved MQ collocation method and the MQ collocation method, the contour lines solved by above two methods with the same shape parameter $c$ are plotted on the $x z$ plane at $y=0.6$ and shown in Fig.5 and Fig.6. It indicates that the improved MQ collocation method gets more accuracy over the traditional MQ method, even to solutions near to the boundary.

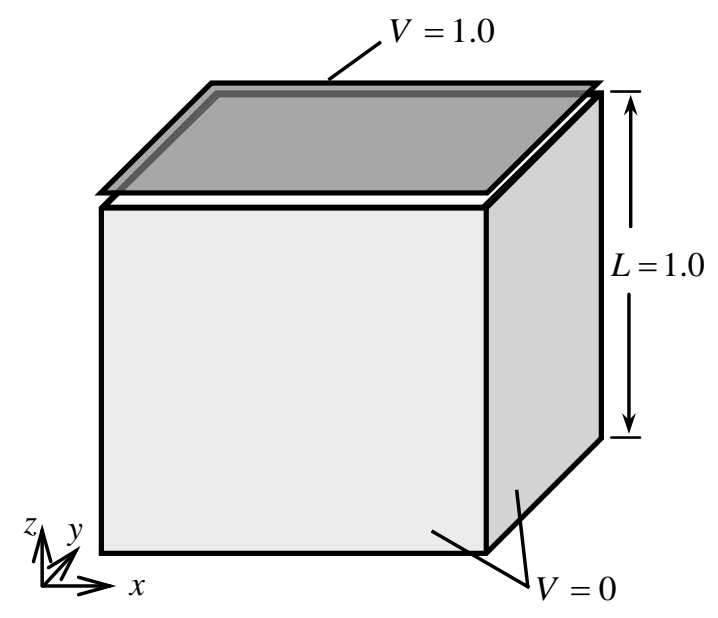

Fig.3 The electrostatic cubical box

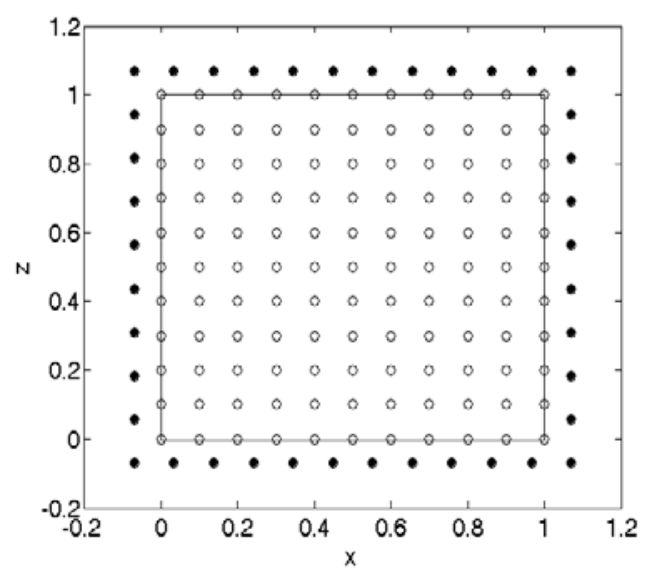

Fig.4 The cross section of the nodes distribution

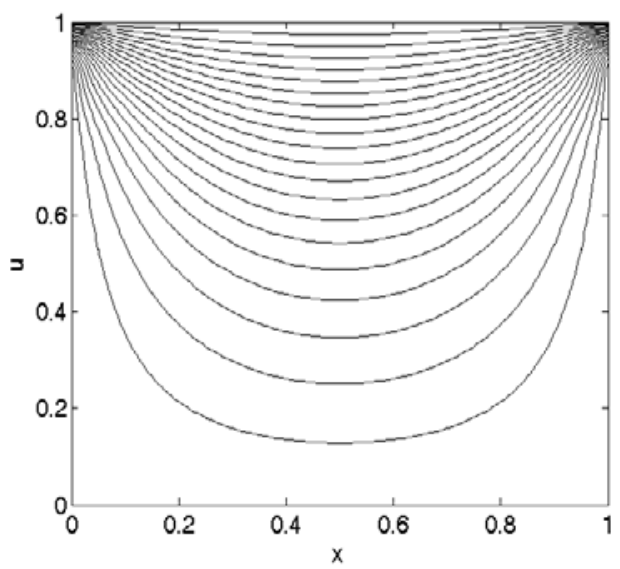

Fig. 5 Potential contours at $\mathrm{y}=0.6$ by improved MQ collocation method 


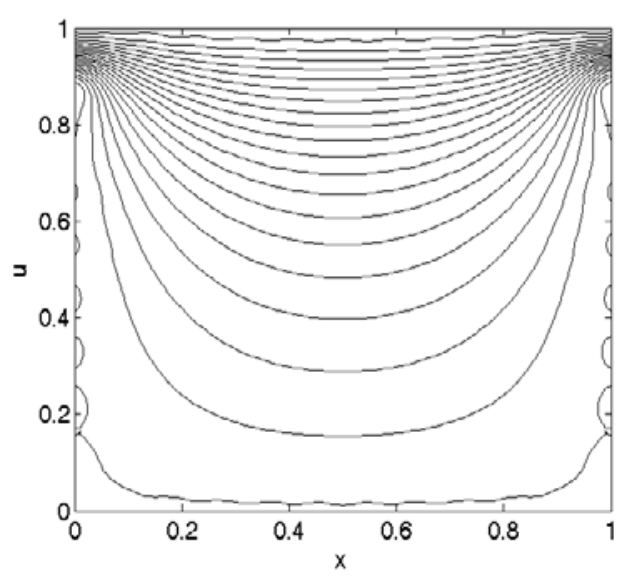

Fig.6 Potential contours at $\mathrm{y}=0.6$ by traditional MQ collocation method

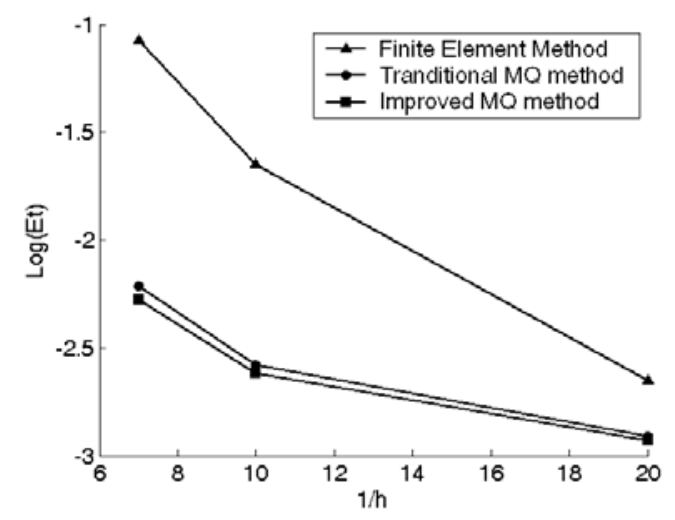

Fig.7 Relative norm error while refining the nodes distribution

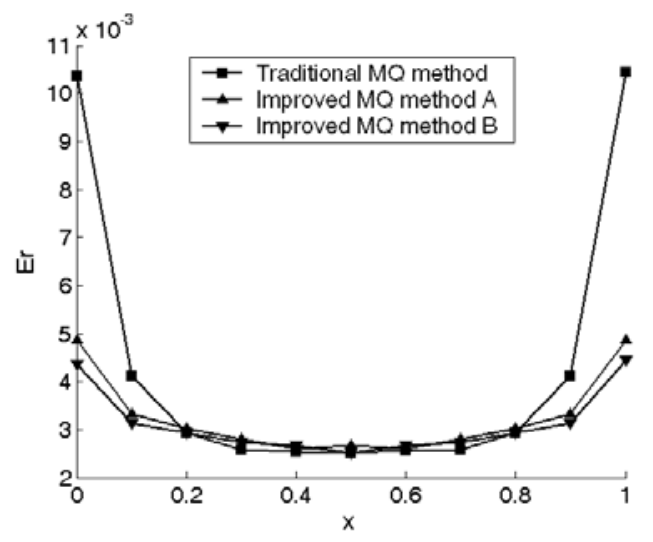

Fig.8 Comparison on relative error limit between MQ methods

In order to illustrate more qualities of $\mathrm{MQ}$ collocation method as a truly meshless method, a comparison on the accuracy between mesh method, such as Finite Element Method (FEM), and the meshless method, as the proposed MQ collocation method, is carried on by error analysis with equation as follows

$$
E t=\frac{1}{N} \sqrt{\sum_{i=1}^{N}(\tilde{u}-u)^{2} / \sum_{i=1}^{N} u^{2}}
$$

Where $N$ is the total number of nodes for solution, $\tilde{u}$ and $u$ are the numerical solution and the analytical solution respectively. Fig.7 shows the relative norm error while refining the nodes distribution. It indicates that MQ collocation methods are much more accurate than FEM within the same node scale $h$, namely, it can get the same level accuracy with fewer nodes or bigger scale $h$, and it is more economical than mesh method as FEM.

Comparison between Fig.5 and Fig.6 shows that the improved MQ collocation method gains more accuracy than the traditional MQ collocation method with the same shape parameter $c$. However, we do not know which is superior to another in the proposed two improved MQ collocation method, in which one is a truly MQ collocation method and another is MQ collocation method by combining with polynomials, and we also do not know the advantages held by the improved MQ collocation method over the traditional MQ collocation method in details. Here we induce a comparison with the relative error limit between these MQ collocation methods as

$$
E r=|\tilde{u}-u| /|u|
$$

Where $\tilde{u}$ and $u$ are the numerical solution and the analytical solution respectively. Fig.8 shows this comparative result, improved MQ collocation method A represents the truly MQ collocation method and improved MQ collocation method B the MQ collocation method by combining with polynomials. We can see the traditional MQ collocation method will result in greater relative error near to the boundary than that resulted by the improved MQ collocation method. However, the MQ collocation method by combining with polynomials does not appears to obviously improve the accuracy for the truly MQ collocation method, although it both increase the complexity of the system matrix and the solving CPU time. This also indicates that the truly MQ collocation method is more economical than the MQ collocation method by combining with polynomials in the same cases.

\section{CONCLUSIONS}

An improved MQ collocation method has been proposed in this paper for 3D electromagnetic problems. The idea of this method is to add an additional set of nodes adjacent to the boundary and, correspondingly, an additional set of collocation equations obtains via collocation of the PED boundary. Results showed that the use of this improved MQ collocation strategy is more accurate than the traditional MQ collocation method and it can obtain the same level accuracy with fewer nodes than mesh method, such as FEM. This method, as a truly meshless method, has very large potential for the solution of much more complex 3D problems.

\section{REFERENCES}

[1] T. Belytschko, et al, "Meshless Methods: An Overview and Recent Developments," Computer Methods in Applied Mechanics and Engineering, Vol.139, pp.3-47, 1996

[2] C. Franke, R. Schaback, "Solving Partial Differential Equations by Collocation Using Radial Basis Functions," Appl. Math. Comp., vol.93, no.1, pp.73-91, 1998.

[3] R. Franke, "Scattered Data Interpolation: Test of Some Method," Math. Comput., vol.48, pp.381-399, 1982.

[4] J.C. Li, Y.C. Hon, C.S. Chen, "Numerical Comparisons of Two Meshless Method Using Radial Basis Functions,” Engng. Anal. Bound. Elem., vlo.26, pp.205-225, 2002

[5] A.I. Fedoseyev, M.J. Fredman, E.J. Kansa, "Improved Multiquadric Method for Elliptic Partial Differential Equations via PDE Collocation on the Boundary," Computers Math. Applic., Vol.43, pp.439-455, 2002 